\title{
THE CORE AND HALO STRUCTURE OF THE QUASAR 4C18.68
}

\author{
Ann C. Gower and J.B.Hutchings \\ University of Victoria and Dominion Astrophysical Observatory \\ Victoria, B.C., Canada.
}

\begin{abstract}
We present new VLA observations of this complex low redshift quasar, which was previously modelled as a precessing twin-jet nucleus. A new $2 \mathrm{~cm}$ map fails to show the predicted curvature near the nucleus. $B$ and $C$ configuration maps have been obtained, to study the halo of the source. These results suggest that the radio source is not very old, and appears to have undergone two major changes of orientation in its $<10^{7}$ year history.
\end{abstract}

\section{RESULTS.}

Figure 1 shows the VLA A-configuration $2 \mathrm{~cm}$ map of the core of the source. This shows a very straight jet from core to bending point and does not conform to the precession model proposed for the $6 \mathrm{~cm}$ and $20 \mathrm{~cm}$ maps ${ }^{1,2}$. The recent history of the source seems to involve a constant orientation.

The halo (Figure 2) reveals a third major orientation of the jet in its outer structure. It appears that the source has always been stronger on one side, and that the jet has undergone successive changes of direction at decreasing intervals of time. In each major orientation there are minor wiggles. The polarisation maps and the spectral index maps also suggest a feature along the centre of the long (SW) axis of the halo. Finally, the differential Faraday rotation (Figure 2) suggests a region of variation of the amount of intervening material along this direction.

The spectral index steepens slightly with distance from the core. For the source size, an equipartition field of $10^{-4} \mathrm{~g}$ is indicated, for which synchrotron loss timescales are $10^{6-7}$ years. There is little correspondence with the optical continuum morphology ${ }^{3}$ or the [O III] tidal tail to the $\mathrm{NE}^{4}$. The scale of the optical tail ( $\sim 30 \mathrm{Kpc}$.) and the distance of the close (interacting?) companions suggest timescales an order of magnitude larger for the formation of the optical structure. However, this does not necessarily rule out a causal connection between the complex optical and radio structures in this quasar.

This work is being published in full elsewhere.

\section{References.}

1. Gower A.C. and Hutchings J.B. 1982, Ap.J. 253 , L1.

2. Gower A.C., Gregory P.C., Hutchings J.B., Unruh W.G. 1982, Ap.J. 262 , 478.

3. Hutchings J.B. et al 1984 Ap.J.Suppl $55,319$.

4. Shara M.M., Moffat A.F.J., Albrecht R. 1985 Ap.J. 296 , 399. 


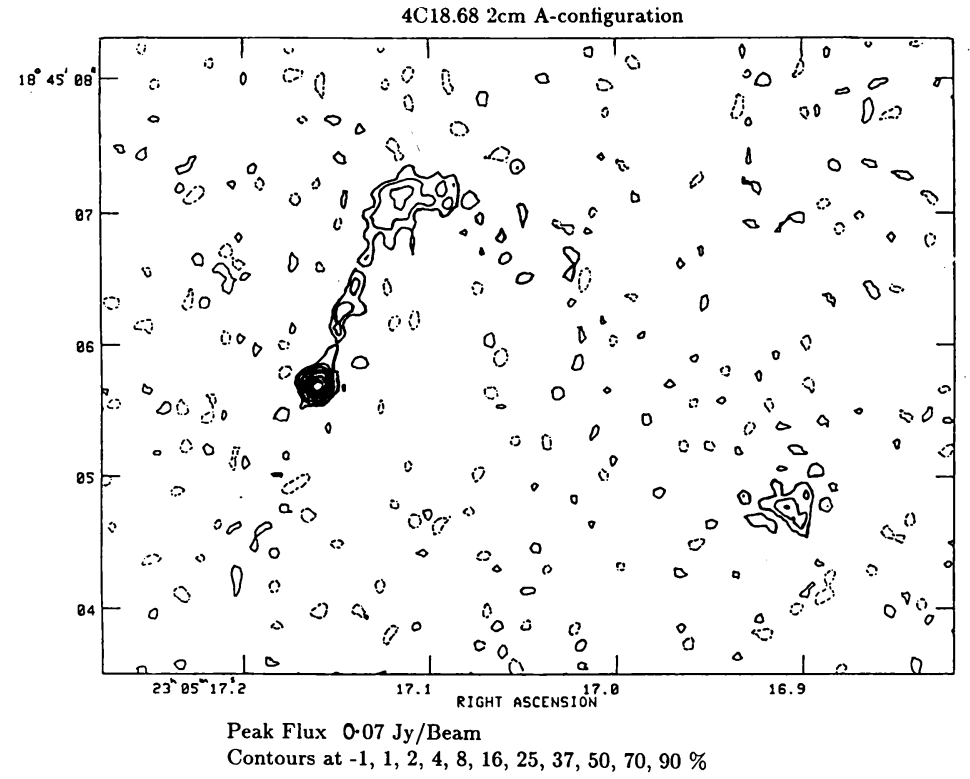

Figure 1. 2cm VLA map of inner structure. Straight jet from core to upper bending point is inconsistent with precession model fitted to longer wavelength maps.
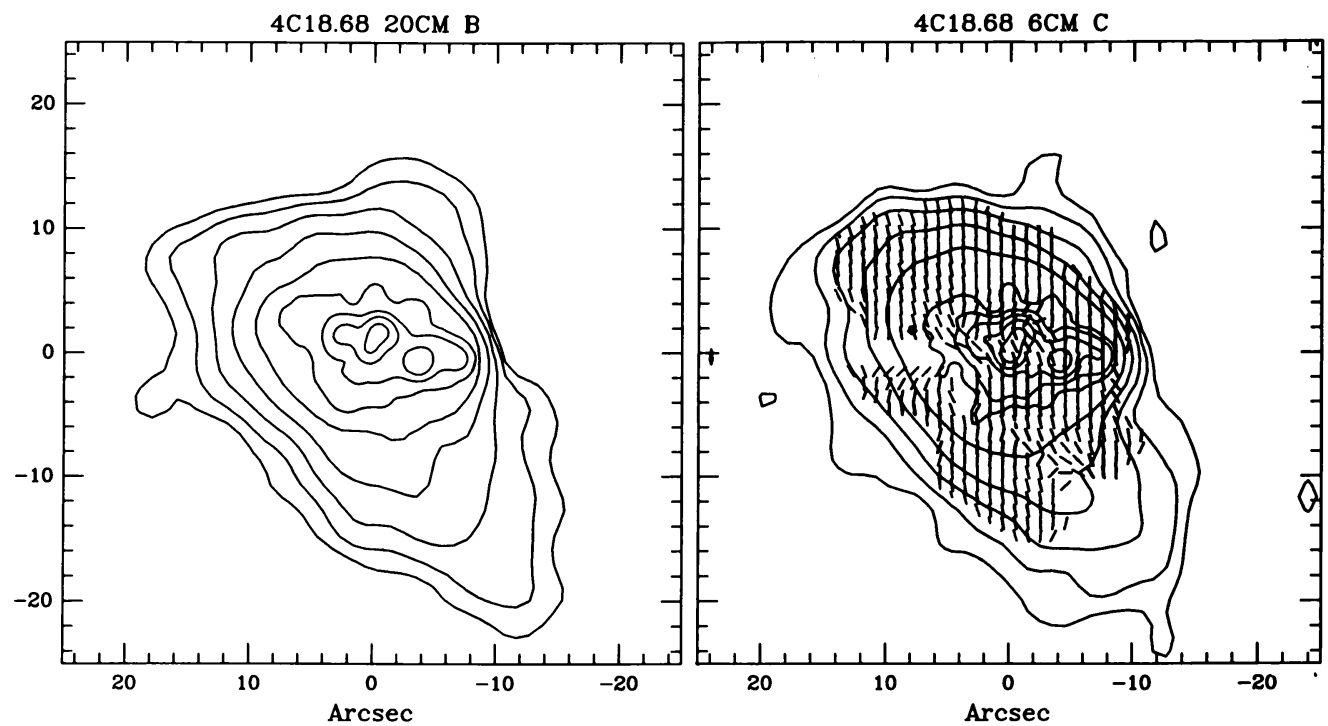

Figure 2. Matched $20 \mathrm{~cm}$ and $6 \mathrm{~cm}$ VLA maps, showing halo, with higher resolution inner region maps. Contours lie a factor 2 apart. Note 3 principal directions of ejection: SW, $\mathrm{S}$ of $\mathrm{W}$, and $\mathrm{NW}$, from outer to inner structure. Vectors in $20 \mathrm{~cm}$ map show differential Faraday rotation, which is generally uniform except for SW outer (earlier jet?) direction, inner bending point, and inner SE region. The latter corresponds to the inner part of the optical tail. 